\title{
A COMMUNICATION FROM THE TREASURER
}

To the Board of Trustees of the American Mathematical Society:

\section{Gentlemen:}

I have the honor to submit herewith a special report of the Treasurer for the six-month period December 1, 1952-May 31, 1953. This is necessitated by the decision of the Trustees to change the fiscal year so as to begin on June 1 and end on May 31 annually instead of running from December 1 to November 30.

Caution should be used in drawing conclusions from the accompanying schedules, as they are not directly comparable with earlier annual reports. It cannot be assumed that figures should normally be approximately half of the analogous figures cited from the annual report dated November 30,1952, as income is not received nor are expenses incurred uniformly throughout the year. Furthermore, the balances on hand include grants for special purposes, largely unexpended, which were not available to the Society in the previous fiscal period.

During the six-month period one $\$ 1000$ Texas and Pacific Railway Company bond was called, $\$ 16,600$ Series G Savings Bonds matured or about to mature were exchanged for the new United States Government $3 \frac{1}{4} \%$ bonds, and $\$ 5,000$ Province of Ontario $3 \frac{1}{4} \%$ bonds were purchased. Also, $\$ 500$ United States Treasury $2 \frac{1}{2}$ 's were sold and $\$ 400$ additional United States $3 \frac{1}{4}$ 's were acquired to round out our holding.

Our return on our invested funds during the six-month period has been at the rate of $4.8 \%$ per annum.

Amortization of the cost of moving to Providence continues as originally scheduled.

Respectfully submitted,

Albert E. Meder, JR.

Treasurer 


\section{EXHIBIT A}

\section{BALANCE SHEET}

Assets

\begin{tabular}{|c|c|c|}
\hline \multicolumn{3}{|l|}{ Assets } \\
\hline & $\begin{array}{c}\text { May 31, } \\
1953\end{array}$ & $\begin{array}{c}\text { November 30, } \\
1952 \\
\text { (adjusted) }\end{array}$ \\
\hline \multicolumn{3}{|l|}{ CURRENT Funds: } \\
\hline \multirow{4}{*}{ 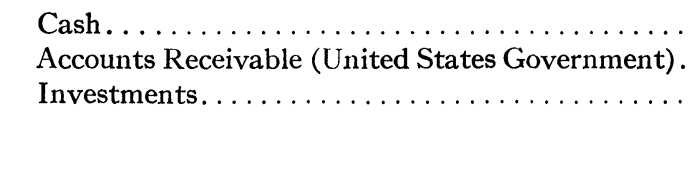 } & $\$ 68,660.23$ & $\$ 19,430.02$ \\
\hline & $5,665.10$ & $4,667.27$ \\
\hline & $67,208.26$ & $67,329.96$ \\
\hline & $\$ 141,533.59$ & $\$ 91,427.25$ \\
\hline \multicolumn{3}{|l|}{ INVESTED FUNDS: } \\
\hline \multirow{3}{*}{ 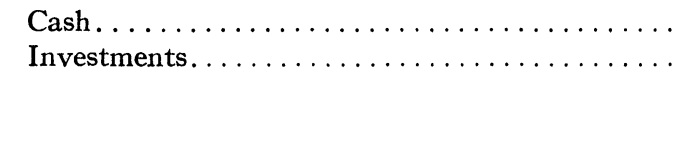 } & $\$ \quad 81.53$ & $\$ 1,109.03$ \\
\hline & $291,892.57$ & $288,090.07$ \\
\hline & $\$ 291,974.10$ & $\$ 289,199.10$ \\
\hline Total Assets. . & $\$ 433,507.69$ & $\$ 380,626.35$ \\
\hline
\end{tabular}

\section{Liabilities}

\section{Current Funds:}

Publications.

$\$ 22,257.16$ $26,610.80$

Other Current Funds.

$2,496.03$

Miscellaneous (Credits).

$90,169.60$

Surplus

$\$ 141,533.59$

$\$(5,042.85)$ $23,918.66$

$1,809.67$ $70,741.77$

$\$ 141,533.59$

$\$ 91,427.25$

\section{INVESTED Funds:}

Endowment Fund Principal. ...............

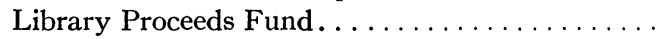

Other Expendable Funds..................

$\$ 94,000.00$

$52,800.00$

$28,551.47$

$\$ 94,000.00$

$50,000.00$

$6,575.00$

$28,551.47$

Prize Funds.

$3,822.48$

$6,575.00$

Life Membership and Subscription Reserve.....

$80,000.00$

$3,822.48$

Mathematical Reviews

$5,550.00$

$80,000.00$

Reserve for Investment Losses...............

Profit/Loss on Sale of Securities.

Total Liabilities 


\section{EXHIBIT B}

\section{GENERAL RECEIPTS AND GENERAL DISBURSEMENTS}

GENERAL RECEIPTS:

Dues from Ordinary Memberships.

$\$ 44,875.28$

Dues from Contributing Memberships.

629.00

Dues from Institutional Memberships.

$5,250.00$

440.00

Publication Charges from Non-Member Institutions. .

45.00

Income From

Henderson Estate. .

$\$ 3,525.00$

Current Funds Investments.

765.74

Invested Funds.

$4,819.61$

$9,110.35$

Miscellaneous

Donations.

$\$ \quad 35.00$

International Congress (Net) ....... $\quad 834.10$

869.10

Overhead on Contracts.

$1,460.71$

Total General Receipts

$\$ 62,679.44$

General Disbursements:

General Activities

Accounting Department

$\$ 4,913.02$

Executive Director.

$10,393.73$

New Haven Office.

$2,440.20$

Officers' Expenses

766.94

Meetings.

$4,135.70$

Policy Committee

134.89

$\$ 22,784.48$

\section{Publications}

Bulletin...

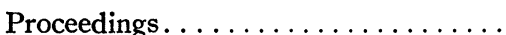

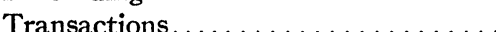

Directory....................

American Journal. . .

$\$ 5,505.96$

$8,521.21$

$2,798.71$

533.88

7.37

Canadian Journal. ............. $1,000.00$

Annals................... 2,100.00

$20,467.13$

Total General Disbursements................ $43,251.61$

Excess of General Receipts over General Disbursements . . . . \$ $\$ 19,427.83$

EXHIBIT C

ANALYSIS OF SURPLUS

Surplus, December $1,1952 \ldots \ldots \ldots \ldots \ldots \ldots \ldots \ldots \ldots \ldots \ldots . \$ 70,741.77$

Excess of General Receipts over General Disbursements..... . 19,427.83

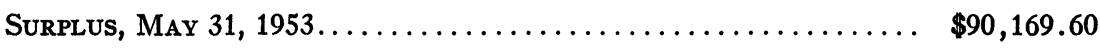




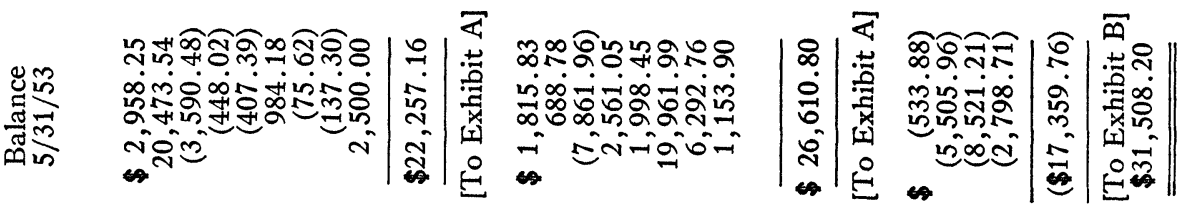
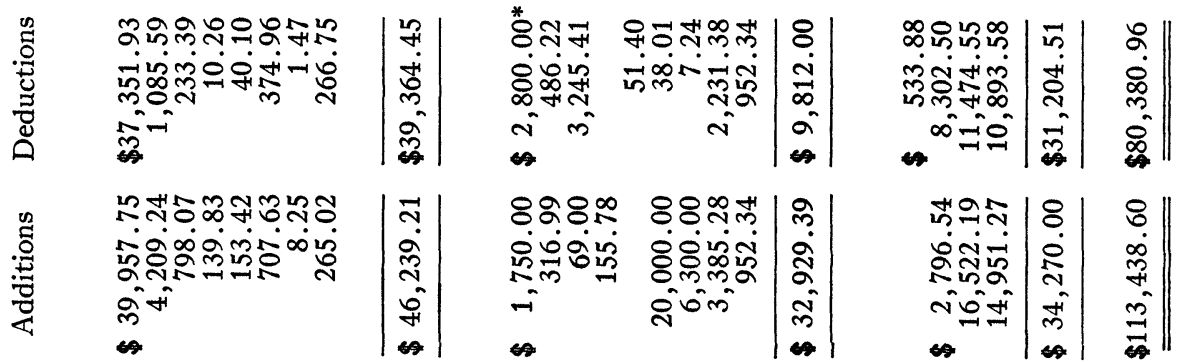

Z
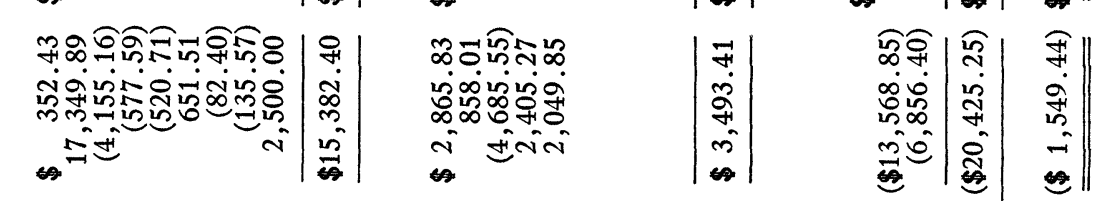

品

II

告

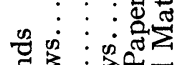

$\left|\begin{array}{c}-1 \\ \dot{q} \\ 2 \\ g \\ \dot{q} \\ m \\ \infty\end{array}\right|$

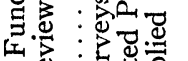

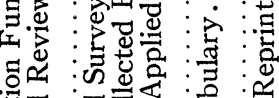

थ

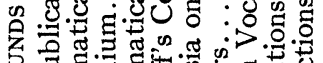

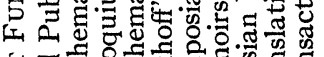

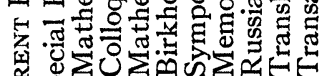

察
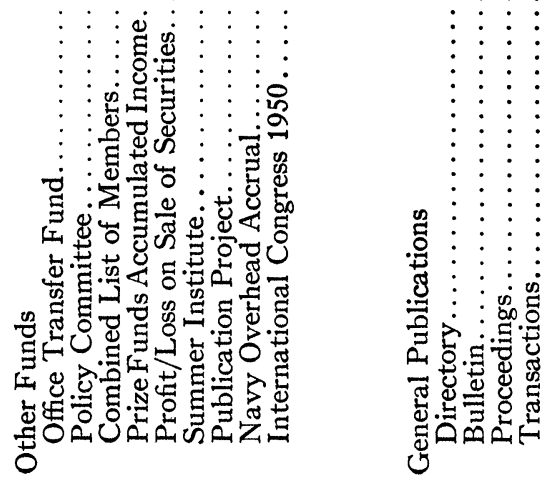


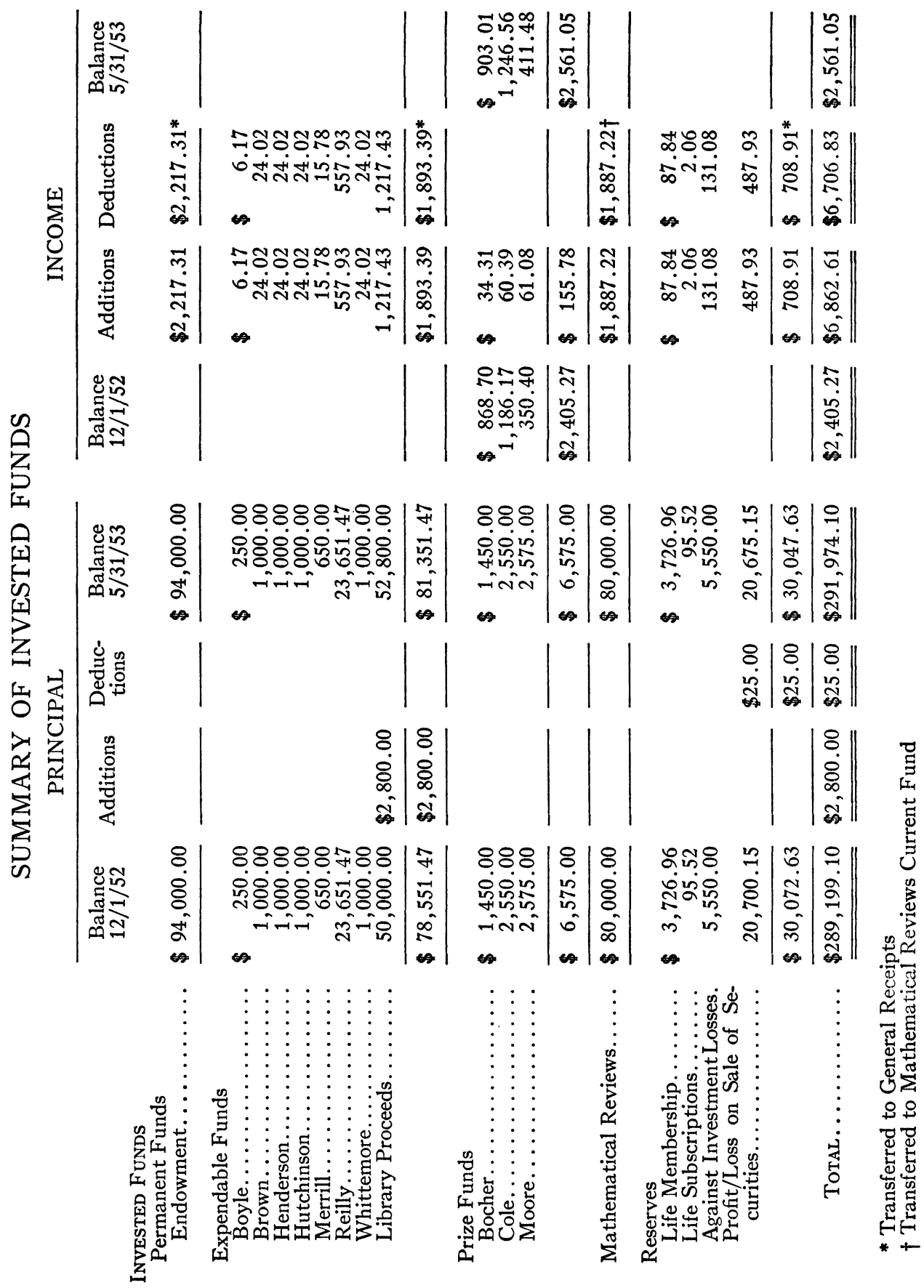

